\title{
BMJ Open Mental health crisis in healthcare providers in the COVID-19 pandemic: a cross-sectional facility-based survey
}

\author{
Chih-Wei Sung, ${ }^{1}$ Chi-Hsin Chen, ${ }^{1}$ Cheng-Yi Fan, ${ }^{1}$ Jia-How Chang, ${ }^{1}$ \\ Chia Chun Hung, ${ }^{2}$ Chia-Ming Fu, ${ }^{3}$ Li Ping Wong, ${ }^{4}$ Edward Pei-Chuan Huang (D) , 1,3 \\ Tony Szu-Hsien Lee (D) ${ }^{5}$
}

To cite: Sung C-W, Chen C-H, Fan C-Y, et al. Mental health crisis in healthcare providers in the COVID-19 pandemic: a cross-sectional facilitybased survey. BMJ Open 2021;11:e052184. doi:10.1136/ bmjopen-2021-052184

- Prepublication history and supplemental material for this paper is available online. To view these files, please visit the journal online. (http://dx.doi. org/10.1136/bmjopen-2021052184).

Received 09 April 2021 Accepted 19 July 2021

Check for updates

(c) Author(s) (or their employer(s)) 2021. Re-use permitted under CC BY-NC. No commercial re-use. See rights and permissions. Published by BMJ.

For numbered affiliations see end of article.

\section{Correspondence to}

Dr Edward Pei-Chuan Huang; edward56026@gmail.com and Professor Tony Szu-Hsien Lee; tonylee@ntnu.edu.tw

\section{ABSTRACT}

Objectives During a pandemic, healthcare providers experience increased mental and physical burden. Burnout can lead to treatment errors, patient mortality, increased suicidal ideation and substance abuse as well as medical malpractice suits among medical staff. This study aimed to investigate the occurrence of burnout, acute stress disorder, anxiety disorder and depressive disorder among healthcare providers at the third month of the COVID-19 pandemic.

Design A cross-sectional facility-based survey.

Setting Hospitals around the country with different levels of care.

Participants A total of 1795 respondents, including 360 men and 1435 women who participated in the survey. Primary outcome measures Burnout was assessed using the Physician Work Life Study. A score of $\geq 3$ implied burnout. Results Of the 1795 respondents, 723 (40.3\%) reported burnout, and 669 (37.3\%) cared for patients with COVID-19. Anxiety levels were mild in $185(10.3 \%)$ respondents, moderate in $209(11.6 \%)$ and severe in $1401(78.1 \%)$. The mean Center for Epidemiologic Studies Depression Scale-10 score was $9.5 \pm 6.3$, and $817(45.5 \%)$ respondents were classified as having depression. Factors associated with burnout were working in acute and critical care (ACC) divisions (adjusted OR $(\mathrm{aOR})=1.84,95 \% \mathrm{Cl} 1.20$ to $3.39, \mathrm{p}=0.019$ ), caring for patients with COVID-19 (aOR=3.90, $95 \% \mathrm{Cl} 1.14$ to $13.37, \mathrm{p}=0.031$ ) and having depressive disorder ( $\mathrm{aOR}=9.44,95 \% \mathrm{Cl} 7.44$ to $11.97, \mathrm{p}<0.001$.

Conclusions Physicians and nurses are vulnerable to burnout during a pandemic, especially those working in ACC divisions. Anxiety disorder, depressive disorder and care of patients with COVID-19 may be factors that influence the occurrence of burnout among healthcare providers.

\section{INTRODUCTION}

The COVID-19 caused by the SARS-CoV-2 was first reported in Wuhan, China, as a novel type of pneumonia. The disease has spread rapidly and internationally since $2020,{ }^{1}$ and the number of confirmed cases increases daily. COVID-19 has spread worldwide in only 1 year. The WHO declared COVID-19 a pandemic disease, and in nearly all countries, medical and social burdens were created,

\section{Strengths and limitations of this study}

- This study is an investigation of the occurrence of burnout among healthcare providers at the early period of the COVID-19 pandemic.

- A population-based snowball sampling technique was employed to collect information rapidly.

- The study comprehensively investigated the mental health of healthcare providers during a disease pandemic

- There was no information on the rejection rate of the respondents.

- Presentations of mental health disorders may vary among countries.

among which was freezing of economic activity and development. ${ }^{2}$

As the fight against the COVID-19 pandemic continues, besides physical health problems, mental health problems, especially among the medical staff, have become a critical concern. During a pandemic, the mental burden on the medical staff increases. Previous studies have demonstrated that an epidemic or pandemic, such as SARS in 2003, significantly influenced mental health, especially in healthcare providers. ${ }^{34} \mathrm{~A} 1$-year follow-up report also indicated that healthcare providers who survived a pandemic had higher severity of mental health disorders and negative symptoms, including burnout, anxiety, depression and acute stress disorder. ${ }^{5}$

Burnout, a state of vital exhaustion, influences health status and healthcare services. It worsens the job performance of medical staff significantly, thereby affecting the patients' safety and health. ${ }^{6-8}$ Burnout also increases the mental load and associated negative emotional symptoms in medical staff. ${ }^{910} \mathrm{~A}$ survey of 270 critical care nurses in the UK revealed a significant negative correlation of -0.24 between burnout and patient safety, ${ }^{6}$ and the results of a meta-analysis indicated 
high levels of burnout in medical staff and worsening of patient safety. ${ }^{8}$ Higher levels of burnout among medical staff may contribute to patients' dissatisfaction with their medical treatment and increase in complaints. ${ }^{11}$

Some studies have focused on the influence of the COVID-19 pandemic on mental health over the past year. ${ }^{12-15}$ However, few studies have reported changes in the mental health among healthcare providers during that time, which were characterised by uncertainty, lack of clarity and lack of safety. Thus, in this study, we investigated the association of burnout with other mental health disorders such as acute stress disorder, anxiety disorder and depressive disorder in healthcare providers during the early stage of the COVID-19 outbreak.

\section{MATERIALS AND METHODS \\ Study design}

We conducted a cross-sectional, web-based anonymous survey with a structured questionnaire. The study protocol was prepared in accordance with the Declaration of Helsinki and the International Conference of Harmonisation-Good Clinical Practice guidelines. The requirement of written consent was waived because the participants were anonymous.

\section{Study setting and respondents}

Healthcare providers aged $\geq 21$ years who were working in all hospitals around Taiwan were included. The population-based snowball sampling technique was used to recruit the respondents from this population. The recruitment started from 12 March to 29 March 2020. We used email, Facebook and LINE (the most popular messaging app in Taiwan) to circulate the survey link among hospital staff. When respondents anonymously completed the survey, we thanked and asked them to disseminate the survey link to their colleagues.

The questionnaire was developed in English (online supplemental file 1) and was then translated into Mandarin. Local experts in the fields of psychology, psychiatry, medicine and public health validated the content of the questionnaire. Prior to this study, the questionnaire was pilot tested.

The purpose of this study was explained to respondents on the first page of the questionnaire, which informed them of the voluntary nature of their participation. Their completion of the questionnaire, which took 5-8 min, implied their consent.

\section{Measures}

The survey consisted of five sections, which assessed (1) demographic background, (2) burnout, (3) acute stress symptoms, (4) anxiety symptoms, and (5) depressive symptoms.

\section{Demographics}

The demographic information concerned respondents' age, sex, education, occupation, hospital division, duration of clinical experience (years) and contact with patients potentially ill with COVID-19. Occupation was categorised as physician, nurse and other medical staff (such as pharmacists and medical radiation technologists). The acute and critical care (ACC) division was defined to cover internal medicine, emergency medicine and anaesthesiology.

\section{Acute stress disorder}

The 19-item version of Acute Stress Disorder Scale (ASDS19), which was based on the criteria in the Diagnostic and Statistical Manual of Mental Disorders, Fourth Edition (American Psychiatric Association, 1994), ${ }^{16}$ consists of four dimensions of symptoms: dissociative, re-experiencing, avoidance and arousal. The ASDS has good sensitivity $(95 \%)$ and specificity $(85 \%)$ for identifying acute stress disorder and good psychometric integrity. ${ }^{16}$ A score of $\geq 9$ in the dissociative dimension combined with a total of $\geq 28$ of the sum of re-experiencing, avoidance and arousal scores was used to define acute stress disorder.

\section{Anxiety disorder}

The 6-item version of State-Trait Anxiety Inventory (STAI-6) was adapted from previous studies ${ }^{17}$ for use in this study. The respondents rated the frequency of experiencing the following six emotional states: calm, tense, upset, relaxed, content and worried. The respondents also rated each anxiety symptom during the COVID-19 pandemic. The sum of the scores ranged from 20 to $80 .{ }^{18}$ Scores of $\leq 37$ indicated no symptoms, 38-44 indicated moderate symptoms and $\geq 45$ indicated severe symptoms. ${ }^{17}$

\section{Depressive disorder}

The Center for Epidemiologic Studies Depression Scale (CES-D) was developed as a 20-item questionnaire. ${ }^{18}$ The 10-item Short Form (CES-D-10) with a 4-point response scale was used to screen for depression. Scores of $\geq 10$ indicated depressive symptoms. ${ }^{19}$ Cronbach's alpha was 0.85 for the study sample.

\section{Outcomes}

ASDS, STAI- 6 and CES-D were translated and validated in Chinese. ${ }^{20-22}$ Burnout, the primary outcome, was defined as a state of fatigue or frustration brought about by devotion to a cause, way of life or relationship that failed to produce the expected reward. ${ }^{23}$ To assess burnout, we used a single item, the Physician Work Life Study, developed with 5404 primary care providers. ${ }^{24}$ This assessment has strong psychometric validity and good sensitivity $(83.2 \%)$ and specificity $(87.4 \%) .{ }^{24}$ In this study, the respondents were asked to rate their level of burnout during the COVID-19 pandemic. Responses were scored on a five-category ordinal scale, and a score of $\geq 3$ implied burnout.

\section{Statistical analysis}

The internal consistency of the items representing the scores was assessed to evaluate the reliability of the assessment scores. Multivariate logistic regression was used to determine the factors influencing burnout. For each independent variable, the OR, $95 \% \mathrm{CI}$ and $\mathrm{p}$ value were calculated. The fit of the regression model was assessed by using 
Table 1 Comparison of demographic characteristics and scores of mental health between non-burnout and burnout groups

\begin{tabular}{|c|c|c|c|c|}
\hline Variables & $\begin{array}{l}\text { Total } \\
(n=1795)\end{array}$ & $\begin{array}{l}\text { Non-burnout } \\
(n=1072)\end{array}$ & $\begin{array}{l}\text { Burnout } \\
(n=723)\end{array}$ & $P$ value \\
\hline Age (years) & $36.7 \pm 8.2$ & $37.0 \pm 8.2$ & $36.3 \pm 8.1$ & 0.094 \\
\hline Sex (male) & $360(20.1)$ & 228 (21.3) & $132(18.3)$ & 0.118 \\
\hline Education & & & & 0.203 \\
\hline High school & $13(0.7)$ & $10(0.9)$ & $3(0.4)$ & \\
\hline College & 1496 (83.3) & 882 (82.3) & $614(84.9)$ & \\
\hline Graduate and above & $286(15.9)$ & $180(16.8)$ & $106(14.7)$ & \\
\hline Occupation & & & & $<0.001$ \\
\hline Doctor & 357 (19.9) & $247(23.0)$ & $110(15.2)$ & \\
\hline Nurse & $1064(59.3)$ & $588(54.9)$ & $476(65.8)$ & \\
\hline Pharmacist & $20(1.1)$ & $8(0.8)$ & $12(1.7)$ & \\
\hline Medical technologist & $93(5.2)$ & $56(5.2)$ & $36(5.1)$ & \\
\hline Medical radiation technologist & $91(5.1)$ & $55(5.1)$ & $36(5.0)$ & \\
\hline Division at hospital & & & & $<0.001$ \\
\hline Internal medicine & 477 (26.6) & $266(24.8)$ & $211(29.2)$ & \\
\hline Surgery & $197(11.0)$ & $111(10.4)$ & 86 (11.9) & \\
\hline Obstetrics and gynaecology & $66(3.7)$ & $46(4.3)$ & $20(2.8)$ & \\
\hline Paediatrics & $82(4.6)$ & $59(5.5)$ & $23(3.2)$ & \\
\hline Emergency medicine & $265(14.7)$ & $142(13.3)$ & $123(17.0)$ & \\
\hline Otolaryngology & $19(1.1)$ & $16(1.5)$ & $3(0.4)$ & \\
\hline Anaesthesiology & $36(2.01)$ & $18(1.68)$ & $18(2.49)$ & \\
\hline Family medicine & $35(2.0)$ & $29(2.7)$ & $6(0.8)$ & \\
\hline Radiology & $85(4.7)$ & $49(4.6)$ & $36(5.0)$ & \\
\hline ACC division & $778(43.3)$ & $426(39.7)$ & $352(48.7)$ & $<0.001$ \\
\hline Seniority (years) & $12.5 \pm 8.0$ & $12.5 \pm 8.2$ & $12.6 \pm 7.8$ & 0.791 \\
\hline Contact with suspicious illness & $669(37.3)$ & $335(31.3)$ & $334(46.2)$ & $<0.001$ \\
\hline ASDS scores & $39.5 \pm 8.0$ & $36.72 \pm 9.0$ & $43.68 \pm 11.2$ & $<0.001$ \\
\hline Dissociative disorder (\%) & $880(49.0)$ & $491(45.8)$ & $389(53.8)$ & $<0.001$ \\
\hline Acute stress disorder (\%) & 595 (33.2) & $290(27.1)$ & $305(42.2)$ & $<0.001$ \\
\hline STAI-6 scores & $55.3 \pm 12.4$ & $51.7 \pm 11.7$ & $60.6 \pm 11.6$ & $<0.001$ \\
\hline Mild anxiety (\%) & $185(10.3)$ & 149 (13.9) & $36(5.0)$ & $<0.001$ \\
\hline Moderate anxiety (\%) & $209(11.6)$ & $158(14.7)$ & $51(7.1)$ & \\
\hline Severe anxiety (\%) & $1401(78.1)$ & 765 (71.4) & $636(88.0)$ & \\
\hline CES-D-10 scores & $9.5 \pm 6.3$ & $6.6 \pm 4.6$ & $13.9 \pm 6.0$ & $<0.001$ \\
\hline Depressive disorder (\%) & $817(45.5)$ & 259 (24.2) & $558(77.2)$ & $<0.001$ \\
\hline
\end{tabular}

ACC, acute and critical care; ASDS, Acute Stress Disorder Scale; CES-D-10, 10-item version of Center for Epidemiologic Studies Depression Scale; STAI-6, 6-item version of State-Trait Anxiety Inventory.

the Hosmer-Lemeshow goodness-of-fit test. ${ }^{25}$ All statistical analyses were performed using SPSS V.20.0 (IBM). A p value $<0.05$ was considered significant.

\section{Patient and public involvement}

There was no public and patient involvement either in the setting of the research questions or the development of the study design.

\section{RESULTS}

A total of 1795 respondents completed the questionnaire during the survey period. The mean age of the respondents was $36.7\left(\mathrm{SD}^{26}=8.2\right)$ years. Most respondents were women and had an education level of college and above. Most respondents were nurses $(\mathrm{n}=1064(59.3 \%))$ or physicians $(\mathrm{n}=357(19.9 \%))$. The mean length of clinical experience was $12.5(\mathrm{SD}=8.0)$ years. The mean ASDS score was $39.5(\mathrm{SD}=8.0)$, and $595(33.1 \%)$ respondents had acute 
Table 2 The association between burnout and risk factors in healthcare providers

\begin{tabular}{|c|c|c|c|c|}
\hline \multirow[b]{2}{*}{ Variables } & \multicolumn{2}{|l|}{ Univariate analysis } & \multicolumn{2}{|l|}{ Multivariate analysis } \\
\hline & OR (95\% Cl) & $P$ value & OR (95\% Cl) & $P$ value \\
\hline ACC division & 1.44 (1.19 to 1.74$)$ & $<0.001$ & 1.30 (1.04 to 1.63$)$ & 0.024 \\
\hline Acute stress disorder & 1.97 (1.61 to 2.40$)$ & $<0.001$ & 0.99 (0.78 to 1.26$)$ & 0.929 \\
\hline \multicolumn{5}{|l|}{ Anxiety } \\
\hline Moderate & 1.34 (0.83 to 2.16$)$ & 0.2387 & 1.36 (0.79 to 2.32$)$ & 0.269 \\
\hline Severe & 3.44 (2.36 to 5.03 ) & $<0.001$ & 1.74 (1.13 to 2.68$)$ & 0.012 \\
\hline Depressive disorder & 10.62 (8.49 to 13.27$)$ & $<0.001$ & 9.44 (7.44 to 11.97$)$ & $<0.001$ \\
\hline
\end{tabular}

ACC, acute and critical care.

stress disorder. The mean total score on the STAI- 6 was $55.3(\mathrm{SD}=12.4) ; 185(10.3 \%)$ respondents were classified as having mild anxiety, $209(11.6 \%)$ as having moderate anxiety and $1401(78.1 \%)$ as having severe anxiety. The mean CES-D-10 score was 9.5 (SD=6.3), and $817(45.5 \%)$ respondents were classified as having depressive disorder. In addition, 723 (40.3\%) respondents reported having burnout. Of the respondents with burnout, a higher percentage worked in the ACC division $(48.7 \%$ vs $39.7 \%$; $\mathrm{p}<0.001$ ). Respondents who reported burnout, compared with those who did not, were more vulnerable to acute stress disorder ( $42.2 \%$ vs $27.1 \%$; $\mathrm{p}<0.001)$, severe anxiety $(88.0 \%$ vs $71.4 \% ; \mathrm{p}<0.001)$ and depressive disorder ( $77.2 \%$ vs $24.2 \%$; $<<0.001$; table 1$)$. The association of burnout with the other study variables is described in table 2. After adjustment for confounders, the respondents who worked in the ACC division, compared with those who did not, had a $33.3 \%$ higher risk of burnout (adjusted OR $(\mathrm{aOR})=1.30,95 \%$ CI 1.04 to $1.63, \mathrm{p}=0.024)$. Members of the medical staff who had ever taken care of patients with suspected COVID-19 had nearly twice the risk of burnout as those who had not $(\mathrm{aOR}=1.87,95 \%$ CI 1.03 to $3.38, p=0.039$ ). Respondents with depressive disorder had nine times greater risk of burnout than those without depressive disorder $(\mathrm{aOR}=9.44,95 \%$ CI 7.44 to $11.97, \mathrm{p}<0.001)$.

Moreover, because working in the ACC division was significantly associated with burnout, the baseline characteristics of respondents in non-ACC were compared with those in ACC divisions (table 3). Of the respondents, 778 (43.3\%) worked in the ACC division, whereas 1017 (56.7\%) did not. Those who worked in the ACC division had higher rates of acute stress disorder $(8.4 \%$ vs $5.5 \%$; $p=0.017)$ and depressive disorder ( $49.4 \%$ vs $42.6 \%$; $p=0.004)$. Overall, nearly half of the respondents in the ACC division reported burnout; this proportion was significantly higher than those in the nonACC divisions $(\mathrm{p}<0.001)$.

In view of the potential interaction of other factors and ACC with burnout, a subgroup analysis was performed with a forest plot (figure 1). In comparison with respondents in the non-ACC divisions, respondents in the ACC division were at a higher risk of burnout if they were female $(\mathrm{aOR}=1.32,95 \%$ CI 1.02 to 1.70$)$, were physicians or nurses (aOR $=1.46,95 \%$ CI 1.13 to 1.88$)$ and had severe anxiety $(\mathrm{aOR}=1.32,95 \%$ CI 1.03 to 1.70$)$. We found no significant interaction between and any other variables.

\section{DISCUSSION}

Over the past few months, most of the publications addressing COVID-19 have focused on the disease and the patients. Although some have addressed mental health in healthcare providers, studies focusing on burnout in the early stage of the pandemic were limited. Thus, our study included a single item to measure burnout, accompanying indices of other emotional disorders in healthcare providers working in hospitals at the outbreak of the COVID-19. The responses help shed light on the risk factors for burnout at a dangerous time during a global outbreak (figure 2). The results indicate that during a pandemic, physicians and nurses are more vulnerable to burnout than other hospital healthcare workers such as pharmacists and medical radiation technologists. The physicians and nurses who serve in the ACC division are clearly at a higher risk of mental exhaustion. Other factors that contributed to burnout included anxiety, depression and contact with patients with COVID-19.

Ide et al mentioned the psychological effects of the COVID-19 pandemic and associated factors on hospital workers at the beginning of the pandemic with the outbreak on the Diamond Princess cruise ship. ${ }^{27}$ The study period of Ide $e t$ al was between the end of March and the beginning of April 2020, which was approximately half a month after ours. Healthcare providers at both high and low risks of infection who worked in hospitals and cared for patients with COVID-19 reported experiencing psychological distress at the beginning of the outbreak. ${ }^{27}$ Feelings of being isolated and of discrimination or COVID-19-related stigmatisation were also investigated..$^{28}$ These feelings represent further stress that contributes to a mental health crisis. 
Table 3 Comparison of demographic characteristics and scores of ASDS, STAI- 6 and CES-D-10 between non-ACC and ACC groups

\begin{tabular}{|c|c|c|c|c|}
\hline Variables & $\begin{array}{l}\text { Total } \\
(n=1795)\end{array}$ & $\begin{array}{l}\text { Non-ACC } \\
(n=1017)\end{array}$ & $\begin{array}{l}\text { ACC } \\
(n=778)\end{array}$ & $P$ value \\
\hline Age (years) & $36.7 \pm 8.2$ & $36.8 \pm 7.9$ & $36.6 \pm 8.5$ & 0.652 \\
\hline Sex (male) & $360(20.1)$ & $182(17.9)$ & $178(22.9)$ & 0.009 \\
\hline Education & & & & $<0.001$ \\
\hline High school & $13(0.7)$ & $10(1.0)$ & $3(0.4)$ & \\
\hline College & $1496(83.3)$ & $825(81.1)$ & $671(86.3)$ & \\
\hline Graduate and above & $286(15.9)$ & $182(17.9)$ & $104(13.4)$ & \\
\hline Occupation & & & & $<0.001$ \\
\hline Doctor & $357(19.9)$ & $174(17.1)$ & $183(23.5)$ & \\
\hline Nurse & $1064(59.3)$ & $529(52.0)$ & $535(68.8)$ & \\
\hline Pharmacist & $20(1.1)$ & $17(1.7)$ & $3(0.4)$ & \\
\hline Medical technologist & $93(5.2)$ & $77(7.6)$ & $16(2.1)$ & \\
\hline Medical radiation technologist & $91(5.1)$ & $81(8.0)$ & $10(1.3)$ & \\
\hline Division at hospital & & & & $<0.001$ \\
\hline Internal medicine & 477 (26.6) & $0(0.0)$ & $477(26.6)$ & \\
\hline Surgery & $197(11.0)$ & $197(11.0)$ & $0(0.0)$ & \\
\hline Obstetrics and gynaecology & $66(3.7)$ & $66(3.7)$ & $0(0.0)$ & \\
\hline Paediatrics & $82(4.6)$ & $82(4.6)$ & $0(0.0)$ & \\
\hline Emergency medicine & $265(14.8)$ & $0(0.0)$ & $265(34.1)$ & \\
\hline Otolaryngology & $19(1.1)$ & $19(1.9)$ & $0(0.0)$ & \\
\hline Anaesthesiology & $36(2.0)$ & $0(0.0)$ & $36(4.6)$ & \\
\hline Family medicine & $35(2.0)$ & $35(3.4)$ & $0(0.0)$ & \\
\hline Radiology & $85(4.7)$ & $85(8.4)$ & $0(0.0)$ & \\
\hline Seniority (years) & $12.5 \pm 8.0$ & $12.4 \pm 8.0$ & $12.7 \pm 8.1$ & 0.382 \\
\hline Contact with suspicious illness & $669(37.3)$ & $241(23.7)$ & $428(55.0)$ & $<0.001$ \\
\hline ASDS scores & $39.5 \pm 8.0$ & $38.9 \pm 10.3$ & $40.4 \pm 10.7$ & 0.004 \\
\hline Dissociative disorder (\%) & $880(49.0)$ & $490(48.2)$ & $390(50.1)$ & 0.413 \\
\hline Acute stress disorder (\%) & $595(33.2)$ & $326(32.1)$ & $269(34.6)$ & 0.261 \\
\hline STAI-6 scores & $55.3 \pm 12.4$ & $54.7 \pm 12.5$ & $56.1 \pm 12.4$ & 0.014 \\
\hline Minimal anxiety (\%) & $185(10.3)$ & $117(11.5)$ & $68(8.7)$ & 0.159 \\
\hline Moderate anxiety (\%) & $209(11.6)$ & $118(11.6)$ & $91(11.7)$ & \\
\hline Severe anxiety (\%) & $1401(78.1)$ & $782(76.9)$ & $619(79.6)$ & \\
\hline CES-D-10 scores & $9.5 \pm 6.3$ & $9.2 \pm 6.3$ & $10.0 \pm 6.4$ & 0.007 \\
\hline Depressive disorder (\%) & $817(45.5)$ & $433(42.6)$ & $384(49.4)$ & 0.004 \\
\hline Burnout (\%) & $723(40.3)$ & $371(36.5)$ & $352(45.2)$ & $<0.001$ \\
\hline
\end{tabular}

ACC, acute and critical care; ASDS, Acute Stress Disorder Scale; CES-D-10, 10-item version of Center for Epidemiologic Studies Depression Scale; STAI-6, 6-item version of State-Trait Anxiety Inventory.

Some studies have focused on the mental health outcomes and associated factors among healthcare providers who treated patients with COVID-19. ${ }^{29-31}$ Lai et al found that front-line healthcare workers were at a higher risk of depression, anxiety, insomnia and distress; however, they did not assess burnout. ${ }^{32}$ Our results show that medical staff in the ACC division had higher incidences of anxiety, depressive disorder and burnout (table 3), but we found no significant difference between male and female healthcare workers. A rational explanation may be that female workers more often expressed symptoms of depression and anxiety. ${ }^{33}$ By contrast, $\mathrm{Wu}$ et al found a lower frequency of burnout among frontline physicians and nurses than among those in standard wards. ${ }^{34}$ A possible reason is that the latter have a heavier workload. The medical staff in ACC units may be more concerned with infection prevention and emotional control in the workplace, factors generally thought to be 


\begin{tabular}{|c|c|c|c|c|c|}
\hline 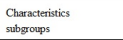 & $\begin{array}{c}\text { Non-ACC } \\
\text { Noof burout }\end{array}$ & $\begin{array}{c}\text { ACC } \\
\text { too of sibicets }\end{array}$ & Adjutatd $O R(95 \%, C)$ & & Intercaction P.-vatu \\
\hline $\begin{array}{l}\text { Sex } \\
\text { Mates } \\
\text { Fenales }\end{array}$ & $\begin{array}{l}601182 \\
3111835\end{array}$ & $\begin{array}{l}72178 \\
2806000\end{array}$ & $\begin{array}{l}1.19(0.70-2.201) \\
1.32(102-1.100)\end{array}$ & 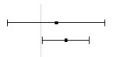 & 0.990 \\
\hline 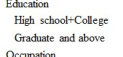 & $\begin{array}{l}3088335 \\
631182\end{array}$ & $\begin{array}{l}3096744 \\
43104\end{array}$ & $\begin{array}{l}1.33(1.04-1.70) \\
0.98(0.51-1.87)\end{array}$ & $\longmapsto$ & 0.759 \\
\hline 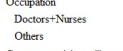 & 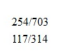 & $\begin{array}{l}332718 \\
2060\end{array}$ & $\begin{array}{l}1.46(1.13-1.189) \\
0.67(0.33-1.38)\end{array}$ & $\mapsto$ & 0.058 \\
\hline 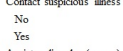 & $\begin{array}{l}255576 \\
116241\end{array}$ & 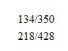 & $\begin{array}{l}1.27(094-1.17) \\
0.97(065-1.44)\end{array}$ & 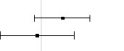 & 0.551 \\
\hline $\begin{array}{l}\text { Ansuedy } \\
\text { No } \\
\text { Yes }\end{array}$ & $\begin{array}{l}49235 \\
322782\end{array}$ & $\begin{array}{l}38159 \\
314619\end{array}$ & 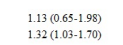 & $\therefore$ & 0.44 \\
\hline $\begin{array}{l}\text { Depese } \\
\text { Nos } \\
\text { Yos }\end{array}$ & $\begin{array}{l}89584 \\
282433\end{array}$ & $\begin{array}{l}76694 \\
276384\end{array}$ & $\begin{array}{l}1.30(093-1.84) \\
1.28(095-1.73)\end{array}$ & $\rightleftarrows$ & 0.899 \\
\hline
\end{tabular}

Figure 1 Subgroup analysis of demographics and variables between the non-acute and critical care (non-ACC) and ACC groups.

drivers of engagement in avoiding burnout. ${ }^{35}$ Because of the pandemic, medical staff in standard wards are now required to take more than the usual preventive measures, ones with which they were not familiar with, including wearing advanced face masks and eye protectors at all times, avoiding group gatherings and immediately reporting their own symptoms to the authorities. These extra burdens can lead to burnout.

The COVID-19 pandemic has far-reaching implications for individuals concerning biological, psychological, and social health and functioning. During the COVID-19 pandemic, major psychological stressors that certainly have increased the risk of burnout include uncertain prognoses; the threat of insufficient medical resources for diagnosis, treatment and prevention; difficulty in shielding healthcare providers from infection because of shortages of personal protective equipment; rapid and enforced imposition of public health policies that infringe on personal freedoms; decreased income; economic recession; and conflicting information from authorities. ${ }^{36}$ Those phenomena occurred early in the COVID-19 pandemic. Burnout in the medical staff may decrease the quality of medical services and affect patient safety, and the fatigue resulting from self-protection and patient care may indirectly cause further spread of COVID-19. A long duration of a pandemic increases the level of burnout in medical staff, contributing to a vicious cycle. Burnout caused by the COVID-19 pandemic has thus seriously affected healthcare providers globally.

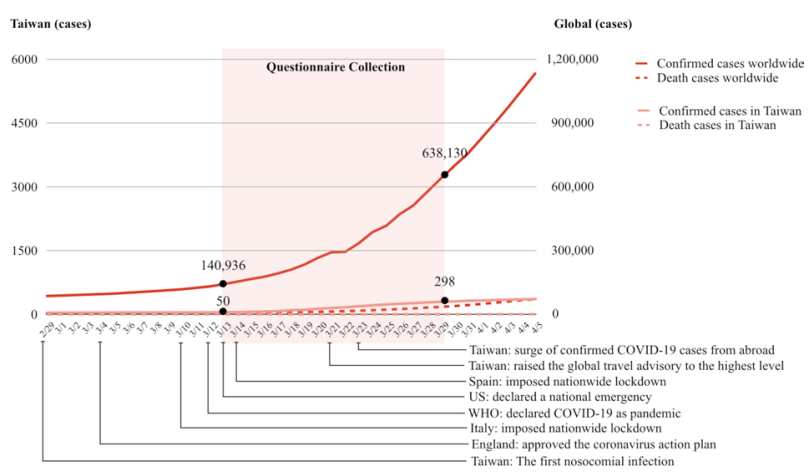

Figure 2 The numbers of confirmed cases and deaths in Taiwan and in the world before, during and after the survey.

\section{Limitations}

This study had some limitations. First, selection bias resulted from the web-based anonymous questionnaire and snowball method used in this study. Although we had emailed all hospital staff through the snowball method, the respondents may not have been a representative population because they completed the survey voluntarily and anonymously. Negative attitudes or symptoms may have been underestimated. Second, selection bias would have influenced the current results. Owing to the snowball sampling design, the investigators had difficulty knowing the total number of eligible recipients. Respondents who were interested in the questionnaire or who believed that the survey could reflect their minds tended to complete the questionnaire. By contrast, those who did not care about their mental health would not complete the questionnaire; thus, the severity of mental health in healthcare providers would be theoretically overestimated. Third, the investigation was not multinational, so the results may not be generalisable to other countries. Fourth, different cut-off values in each inventory may have existed in different populations such as by countries or ethnicities. Finally, potential measurement bias may occur. Burnout, as well as other mental health indices, may be correlated with the severity of the COVID-19 outbreak in different countries; burnout in Taiwan may have been less severe than that in other countries.

\section{CONCLUSIONS}

Physicians and nurses are vulnerable to burnout during the COVID-19 pandemic, especially those in the ACC division. Anxiety disorder, depressive disorder and care of patients with COVID-19 may contribute to burnout in healthcare providers.

\section{Author affiliations}

${ }^{1}$ Department of Emergency Medicine, National Taiwan University Hospital Hsin-Chu Branch, Hsinchu City, Taiwan

${ }^{2}$ Bali Psychiatric Center, Ministry of Health and Welfare, New Taipei City, Taiwan ${ }^{3}$ Department of Emergency Medicine, National Taiwan University Hospital, Taipei City, Taiwan

${ }^{4}$ Department of Social and Preventive Medicine, Faculty of Medicine, University of Malaya, Kuala Lumpur, Malaysia

${ }^{5}$ Department of Health Promotion and Health Education, National Taiwan Normal University, Taipei City, Taiwan

Acknowledgements We appreciate our respondents taking the time to complete the survey during their busy and intensive work schedules. We thank the staff of the Department of Medical Research, National Taiwan University Hsin-Chu Hospital for their assistance in the statistical analyses.

Contributors EPCH and TSHL conceived the main study. CWS and CHC conceived the primary analysis. CWS, EPCH and TSHL designed the study. CYF performed the data extraction. CWS and CYF performed the statistical analyses. JHC, $\mathrm{CCH}$ and CMF contributed to the interpretation of results. CWS drafted the manuscript, which LPW, EPCH and TSHL contributed to, revised critically and approved.

Funding The study was subsidised by the National Taiwan Normal University (202003HS002) and by the National Taiwan University Hospital Hsin-Chu Branch (110-HCH019).

Competing interests None declared.

Patient consent for publication Not required. 
Ethics approval The Research Ethics Committee of the National Taiwan Normal University (No 202003HS002) reviewed and approved the study protocol.

Provenance and peer review Not commissioned; externally peer reviewed.

Data availability statement № additional data are available.

Supplemental material This content has been supplied by the author(s). It has not been vetted by BMJ Publishing Group Limited (BMJ) and may not have been peer-reviewed. Any opinions or recommendations discussed are solely those of the author(s) and are not endorsed by BMJ. BMJ disclaims all liability and responsibility arising from any reliance placed on the content. Where the content includes any translated material, BMJ does not warrant the accuracy and reliability of the translations (including but not limited to local regulations, clinical guidelines, terminology, drug names and drug dosages), and is not responsible for any error and/or omissions arising from translation and adaptation or otherwise.

Open access This is an open access article distributed in accordance with the Creative Commons Attribution Non Commercial (CC BY-NC 4.0) license, which permits others to distribute, remix, adapt, build upon this work non-commercially, and license their derivative works on different terms, provided the original work is properly cited, appropriate credit is given, any changes made indicated, and the use is non-commercial. See: http://creativecommons.org/licenses/by-nc/4.0/.

ORCID iDs

Edward Pei-Chuan Huang http://orcid.org/0000-0002-4800-2561

Tony Szu-Hsien Lee http://orcid.org/0000-0003-2574-3372

\section{REFERENCES}

1 Chen SY, Lee YL, Lin YC. Multicenter evaluation of two chemiluminescence and three lateral flow immunoassays for the diagnosis of COVID-19 and assessment of antibody dynamic responses to SARS-CoV-2 in Taiwan. Emerg Microbes Infect;2020:1-60.

2 L'Angiocola PD, Monti M. COVID-19: the critical balance between appropriate governmental restrictions and expected economic, psychological and social consequences in Italy. are we going in the right direction? Acta Biomed 2020;91:35-8.

3 Chua SE, Cheung V, Cheung C, et al. Psychological effects of the SARS outbreak in Hong Kong on high-risk health care workers. Can J Psychiatry 2004;49:391-3.

4 Huang C-T, Sung C-W, Chen $\mathrm{C}-\mathrm{H}$, et al. The impact of previous epidemic disease on mental health in physicians and nurses during COVID-19 pandemic. Am J Emerg Med 2021. doi:10.1016/j. ajem.2021.01.082. [Epub ahead of print: 02 Feb 2021].

5 Lee AM, Wong JGWS, McAlonan GM, et al. Stress and psychological distress among SARS survivors 1 year after the outbreak. Can J Psychiatry 2007;52:233-40.

6 Al Ma'mari Q, Sharour LA, Al Omari O. Fatigue, burnout, work environment, workload and perceived patient safety culture among critical care nurses. Br J Nurs 2020;29:28-34.

7 Davis D. Nursing burnout and patient safety. JAMA 2003;289:549.

8 Garcia CdeL, Abreu LCde, Ramos JLS, et al. Influence of burnout on patient safety: systematic review and meta-analysis. Medicina 2019;55. doi:10.3390/medicina55090553. [Epub ahead of print: 30 Aug 2019].

9 Aiken LH, Sloane D, Griffiths P, et al. Nursing skill mix in European hospitals: cross-sectional study of the association with mortality, patient ratings, and quality of care. BMJ Qual Saf 2017;26:559-68.

10 Van Gerven E, Vander Elst T, Vandenbroeck S, et al. Increased risk of burnout for physicians and nurses involved in a patient safety incident. Med Care 2016;54:937-43.

11 Profit J, Sharek PJ, Amspoker AB, et al. Burnout in the NICU setting and its relation to safety culture. BMJ Qual Saf 2014;23:806-13.

12 Görlich Y, Stadelmann D. Mental health of flying cabin crews: depression, anxiety, and stress before and during the COVID-19 pandemic. Front Psychol 2020;11:581496.
13 Lopez Steinmetz LC, Dutto Florio MA, Leyes CA. Levels and predictors of depression, anxiety, and suicidal risk during COVID-19 pandemic in Argentina: the impacts of quarantine extensions on mental health state. Psychol Health Med;2020:1-17.

14 Liu CH, Zhang E, Wong GTF, et al. Factors associated with depression, anxiety, and PTSD symptomatology during the COVID-19 pandemic: clinical implications for U.S. young adult mental health. Psychiatry Res 2020;290:113172.

15 Lasalvia A, Amaddeo F, Porru S, et al. Levels of burn-out among healthcare workers during the COVID-19 pandemic and their associated factors: a cross-sectional study in a tertiary hospital of a highly burdened area of north-east Italy. BMJ Open 2021;11:e045127.

16 Bryant RA, Moulds ML, Guthrie RM. Acute stress disorder scale: a self-report measure of acute stress disorder. Psychol Assess 2000;12:61-8.

17 Marteau TM, Bekker H. The development of a six-item short-form of the state scale of the Spielberger State-Trait anxiety inventory (STAI). Br J Clin Psychol 1992;31:301-6.

18 Radloff L. The CES-D scale: a self-report depression scale for research in the general population. Appl Psychol Meas 1977;1:385-401.

19 Andresen EM, Malmgren JA, Carter WB, et al. Screening for depression in well older adults: evaluation of a short form of the CES-D (center for epidemiologic studies depression scale). Am J Prev Med 1994;10:77-84.

20 Lee KL, YL O, Chen S-H. The psychometric properties of a short form of the CES-D used in the Taiwan longitudinal study on aging. $J$ Formosa Ment Health 2009;22:383-410.

21 Shek DT. The Chinese version of the State-Trait anxiety inventory: its relationship to different measures of psychological well-being. J Clin Psychol 1993;49:349-58.

22 Lu CH, Kung YW, Su YJ. Psychometric properties of the Chinese version of Acute Stress Disorder Scale. In: Poster session presented at the 4th Asian Congress of health psychology. Taipei, Taiwan, 2010.

23 Durand-Moreau QV. Is burn-out finally a disease or not? Occup Environ Med 2019;76:938.

24 Dolan ED, Mohr D, Lempa M, et al. Using a single item to measure burnout in primary care staff: a psychometric evaluation. J Gen Intern Med 2015;30:582-7.

25 Paul P, Pennell ML, Lemeshow S. Standardizing the power of the Hosmer-Lemeshow goodness of fit test in large data sets. Stat Med 2013;32:67-80.

26 Teasdale G, Maas A, Lecky F, et al. The Glasgow coma scale at 40 years: standing the test of time. Lancet Neurol 2014;13:844-54.

27 Ide K, Asami T, Suda A, et al. The psychological effects of COVID-19 on hospital workers at the beginning of the outbreak with a large disease cluster on the diamond Princess cruise SHIP. PLoS One 2021;16:e0245294.

28 Mostafa A, Sabry W, Mostafa NS. COVID-19-related stigmatization among a sample of Egyptian healthcare workers. PLoS One 2020;15:e0244172:12.

29 Proto E, Quintana-Domeque C. COVID-19 and mental health deterioration by ethnicity and gender in the UK. PLoS One 2021;16:e0244419.

30 Gloster AT, Lamnisos D, Lubenko J, et al. Impact of COVID-19 pandemic on mental health: an international study. PLoS One 2020;15:e0244809:12.

31 Newby JM, O'Moore K, Tang S, et al. Acute mental health responses during the COVID-19 pandemic in Australia. PLoS One 2020;15:e0236562.

32 Lai J, Ma S, Wang Y, et al. Factors associated with mental health outcomes among health care workers exposed to coronavirus disease 2019. JAMA Netw Open 2020;3:e203976.

33 Altemus M, Sarvaiya N, Neill Epperson C. Sex differences in anxiety and depression clinical perspectives. Front Neuroendocrinol 2014;35:320-30.

34 Wu Y, Wang J, Luo C, et al. A comparison of burnout frequency among oncology physicians and nurses working on the frontline and usual wards during the COVID-19 epidemic in Wuhan, China. J Pain Symptom Manage 2020;60:e60-5.

35 Shanafelt TD, Noseworthy JH. Executive leadership and physician well-being: nine organizational strategies to promote engagement and reduce burnout. Mayo Clin Proc 2017;92:129-46.

36 Pfefferbaum B, North CS. Mental health and the Covid-19 pandemic. N Engl J Med 2020;383:510-2. 